\title{
CLOSURE WAVES IN HELICAL COMPRESSION SPRINGS WITH INELASTIC COIL IMPACT ${ }^{1}$
}

\author{
BY \\ J. A. MORRISON ${ }^{2}$ \\ Brown University
}

Summary. This paper deals with the problem of spring surges taking into account coil closure. This may occur in many cases of compression springs subject to impact. Inelastic coil on coil impact conditions are assumed. The simple theory of spring surges is adopted wherein only the motion of the spring wire parallel to the axis of the spring is considered and the assumption made that each element of the spring satisfies the forcelongitudinal strain relation of the whole spring, before coil closure occurs. The basic theory of coil closure with inelastic impact conditions has been given by Lee [1] ${ }^{3}$. The spring is initially at rest and unstrained with one end fixed and the other (the impact end) is given an impulsive velocity and then either maintained at this velocity or decelerated at a constant rate. The case of a mass attached to the impact end is also considered. Conditions are obtained for which partial or complete closure of the spring occurs.

1. Introduction. We are concerned with the motion of a helical compression spring as depicted diagramatically in Fig. 1, the end $x=l$ being fixed. Following [1] we use as



FIG. 1.

an independent space variable the position $x$ along the unstrained spring. The displacement of an element of the spring in position $x$ is taken as $u(x, t)$, so that the position of the element at time $t$ is given by $(x+u)$. If $f$ is the compressive force transmitted

${ }^{1}$ Received March 13, 1953. The results presented in this paper were obtained in the course of research sponsored by the Ballistic Research Laboratories, Aberdeen Proving Ground under contract DA-19020-ORD-798 with Brown University.

2Research Assistant, Graduate Division of Applied Mathematics, Brown University.

${ }^{3}$ Numbers in square brackets refer to the Bibliography at the end of the paper. 
across the section $x$, then the equation of motion of an element $d x$ of the spring is

$$
u_{t t}=-\frac{1}{m} f_{x},
$$

where $m$ is the mass of the spring per unit original length. This theory is analogous with that of longitudinal waves in a linear elastic material and here $f$ replaces the nominal stress and $m$ the density. The nominal compressive strain of an element of the spring is $\epsilon=-u_{z}$. Before closure occurs there is a linear relation between the stress and the strain given by

$$
f=E \epsilon,
$$

where $E$ is a constant depending on the dimensions and material of the spring.

Substituting in (1) gives the linear wave equation for $u$, .

$$
u_{t t}-c_{0}^{2} u_{x x}=0,
$$

where $c_{0}=\sqrt{E / m}$ is the constant velocity of wave propagation.

The velocity $v$ of an element of the spring is given by $v=u_{t}$. The characteristics in the $(x, t)$-plane are the straight lines $\left(x \pm c_{0} t\right)=$ constant. The corresponding characteristic relations are $c_{0} \epsilon \mp v=$ constant, or $m c_{0} v \mp f=$ constant.

Now let us introduce the dimensionless variables

$$
T=\frac{c_{0}}{l} t ; \quad X=\frac{x}{l} ; \quad V=\frac{v}{v_{0}} ; \quad \Sigma=\frac{f}{f_{0}} ; \quad \Phi=\frac{\epsilon}{\epsilon_{0}},
$$

where $l$ is the length of the unstrained spring, $v_{0}$ is the initial impulsive velocity of the impact end, $f_{0}=m c_{0} v_{0}$ and $\epsilon_{0}=v_{0} / c_{0}$.

Then the characteristic lines in the $(X, T)$-plane are

$$
X \pm T=\text { constant, }
$$

and the corresponding characteristics relations are

$$
V \mp \Sigma=\text { constant, or } V \mp \Phi=\text { constant. }
$$

We now consider closure of the coils. This takes place when the approach of sections of adjacent coils with the same angular position is equal to the initial separation of the coils. This condition is expressed analytically in the form

$$
u(x)-u(x+p)=p-d,
$$

where $p$ is the pitch of the coils and $d$ is their thickness.

Since we are considering in this paper only inelastic coil impact conditions so that the coils remain in contact after closure (until separated by the elasticity of the spring) and we are concerned only with the average effects around a single coil it is permissible to replace the finite difference condition in (6) by a derivative (see [1] for a fuller discussion) so that coil closure is to be associated with a fixed upper bound for the compressive strain $\epsilon$, giving the condition

$$
\epsilon=-u_{x} \leq(p-d) / p=\epsilon_{\max } .
$$


or

$$
\Phi \leq K
$$

where $K=\epsilon_{\max } / \epsilon_{0}$. Hence we have

$$
\begin{array}{lll}
\Phi=\Sigma, & \text { for } & \Sigma \leq K \\
\Phi=K, & \text { for } & \Sigma>K
\end{array}
$$

since an increase in the compressive force may take place when coil closure has occurred but no increase can take place in $\Phi$ according to (8).

The relation between $\Sigma$ and $\Phi$ is shown in Fig. 2. Considerations of continuity and

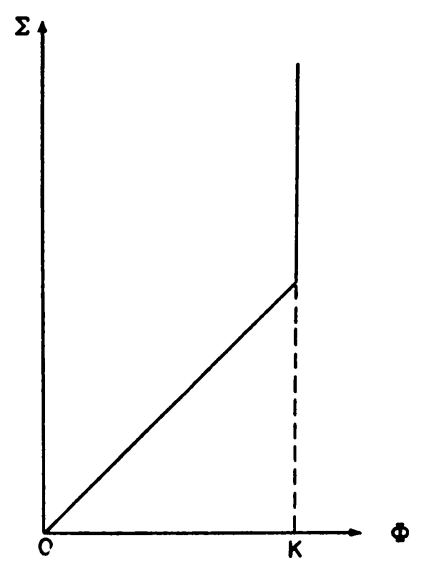

FIG. 2.

momentum across wave fronts of closure yield the conditions

$$
\left.\begin{array}{rl}
|\Delta v| & =c \cdot \Delta \epsilon \\
\Delta f & =m c \cdot|\Delta v|
\end{array}\right\}
$$

where $\Delta$ represents the discontinuity of the variable due to the passage of the wave, and $c$ is the magnitude of the wave velocity, relative to the unstrained spring.

In terms of dimensionless variables Eq. (10) becomes

$$
\begin{aligned}
|\Delta V| & =\Omega \cdot \Delta \Phi \\
\Delta \Sigma & =\Omega \cdot|\Delta V|
\end{aligned}
$$

where $\Omega=c / c_{0}$. Equations (4), (5), (9) and (11) are used in determining the solution of the problem for prescribed boundary conditions.

2. Impulsive velocity at impact end maintained. In this section we consider the case in which the end $x=0$ is given an impulsive velocity $v_{0}$ at time $t=0$ and this velocity is maintained, until complete closure of the spring has taken place. The spring is taken to be initially unstrained and at rest. Until closure occurs the configuration in 
the $(X, T)$-plane is as shown in Fig. $3, X=0$ corresponding to the impact end and $X=1$ to the fixed end of the spring. Throughout the motion $W=\left.V\right|_{z=0}=1$.

A wave of velocity, stress and strain discontinuity is propagated along the spring with constant speed $c_{0}$, commencing at the impact end at time $t=0$ and being reflected alternatively at the ends. Using Eqs. (4) and (5) we find that

$$
V=\frac{1}{2}\left\{1-(-1)^{r}\right\}, \quad \Sigma=r \quad(r \geq 0),
$$



Fig. 3.

in region $r$ of the $(X, T)$-plane. It is seen that after the passage of the discontinuity wave front the stress at a certain position in the spring is increased by an amount $f_{0}$ and remains unchanged until the wave again passes. The corresponding velocity is alternatively $v_{0}$ and 0 .

Now closure does not occur so long as $\Sigma<K$. Since $\Sigma=r$ in region $r$ it follows that closure must eventually occur, as is otherwise evident. Further, when closure commences it does so from an end of the spring.

Let $n<K \leq n+1$ ( $n \geq 0$, integer). Then closure eommences at $T=n$ and from the impact end if $n$ is even, from the fixed end if $n$ is odd. We first consider the case when $n$ is even. Since we are assuming inelastic coil impact conditions the coils do not separate after closure so that at $T=n+\tau$ a portion of the spring $0 \leq X \leq \chi$ is moving as a 
rigid body with uniform speed equal to that of the impact end. It follows that the stress throughout this portion is constant.

Now the coils in front of the closure wave are at rest and the stress is given by $\Sigma=n$. Hence in (11) we have $|\Delta V|=1$, and $\Delta \Phi=(K-n)$, using (8) and (9). Also $\Omega=d \chi / d \tau$. So from (11) we obtain

$$
\Omega=1 /(K-n), \quad \Delta \Sigma=1 /(K-n) .
$$

Hence the closure wave velocity is constant as shown in Fig. 4(a) (for the case $n=2$ ).

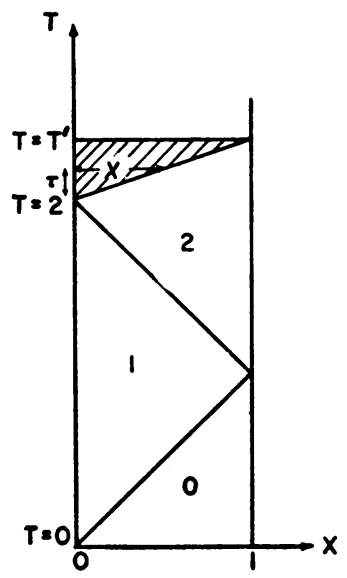

(o)

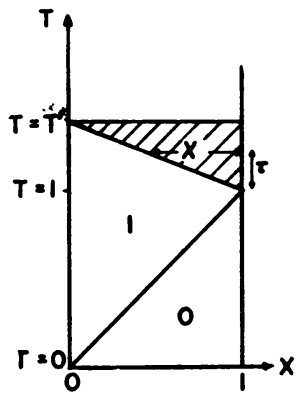

(b)

FIG. 4.

The shaded portion depicts coil closure. Complete closure of the spring has taken place at $\tau=(K-n)$, and then $T=T^{\prime}=K$.

The stress at $X=\chi$ is given by $n+\Delta \Sigma=\{n+1 /(K-n)\}$. If $K$ is close to $n$ this stress is large, but so also is $\Omega$. (Note $\Omega \geq 1$ ). We have in fact,

$$
\begin{aligned}
\int_{T=n}^{T-K} \Delta f d t & =f_{0} \frac{l}{c_{0}} \int_{T=n}^{K} \Delta \Sigma d T=\frac{l f_{0}}{c_{0}}=m l v_{0} \\
& =\text { (weight of spring) } \times \text { (impact velocity) } .
\end{aligned}
$$

When $n$ is odd closure commences from the fixed end. Ahead of the closure wave the stress is given by $\Sigma=n$ and the velocity is $v_{0}$, so $V=1$. In this case the closed portion is at rest, so again we have $|\Delta V|=1$ and $\Delta \Phi=(K-n)$. Hence the closure wave velocity is given by $\Omega=1 /(K-n)$, as before, and the stress in the closed portion is again $\{n+1 /(K-n)\}$.

The $(X, T)$-plane is shown in Fig. $4(\mathrm{~b})$ for the case $n=1$.

3. Impact with constant retardation. In this section we consider the case in which the end $x=0$ is retarded at a uniform rate $a$, after being given an impulsive velocity $v_{0}$ at time $t=0$. The spring is again taken to be initially unstrained and at rest. Until closure occurs the configuration in the $(X, T)$-plane is as shown in Fig. 3. During the motion the velocity at the impact end is given by $W=(1-\lambda T)$, where $\lambda=a l / c_{0} v_{0}>0$. 
As in the previous case a wave of velocity, stress and strain discontinuity is propagated along the spring with constant speed $c_{0}$, commencing at time $t=0$ from the impact end and being reflected alternately at the ends. By making use of Eqs. (4) and (5) we find expressions for $V$ and $\Sigma$ as given in the Appendix. We find that, if closure does occur, then it takes place from an end of the spring and moreover from that end at which reflection of the wave of discontinuity has just occurred.

We next investigate the time at which closure occurs, if at all. Let $\Sigma^{*}$, denote the value of $\Sigma$ at $T=(r-1)+0,(r \geq 1)$, at the end $X=0$ if $r$ is odd and at the end $X=1$ if $r$ is even.

Then

$$
\left\{\begin{array}{l}
\Sigma_{2 k+1}^{*}=\left\{(2 k+1)-2 k^{2} \lambda\right\} \\
\Sigma_{2 k+2}^{*}=2(k+1)(1-k \lambda)
\end{array}\right\} \quad k \geq 0 .
$$

In particular, $\Sigma_{1}^{*}=1, \Sigma_{2}^{*}=2$, i.e. independent of $\lambda$. Closure occurs when $\Phi=\Sigma=$ $K(0<K<\infty)$. Therefore, if $0<K \leq 1$, closure commences immediately from the impact end and if $1<K \leq 2$ from the fixed end after the passage of the first wave. These results are independent of $\lambda$, so we now need consider only $2<K<\infty$. The case $\lambda=0$ was discussed in the previous section and it was shown that closure always took place.

Case $\lambda \geq 1 / 2$.

Here $\Sigma_{r}^{*} \leq 2,(r \geq 1)$, since

$$
\left(\Sigma_{2 k+2}^{*}-\Sigma_{2 k+1}^{*}\right)=\left(\Sigma_{2 k+1}^{*}-\Sigma_{2 k}^{*}\right)=(1-2 k \lambda), \quad(k \geq 1) .
$$

Hence if $K>2$ no closure occurs.

Case $\quad \frac{1}{2(p+1)} \leq \lambda<\frac{1}{2 p}, p \geq 1, \quad$ integer.

Here $\Sigma_{r}^{*}$ increases strictly up to $\Sigma_{2 p+2}^{*}$ and $\Sigma_{r}^{*} \leq \Sigma_{2 p+2}^{*}$, all $r$. [See (12)].

But $\Sigma_{2 p+2}^{*}=2(p+1)(1-p \lambda)$. Hence, if $\bar{K}>2(p+1)(1-p \lambda)$, no closure occurs. If $2<K \leq 2(p+1)(1-p \lambda)$, then closure does occur. In this case there is an integer $q$ such that $2 \leq q \leq(2 p+1)$ and $\Sigma_{a}^{*}<K \leq \Sigma_{a+1}^{*}$. Hence closure commences a $T=q+0$, and from the impact end if $q$ is even, from the fixed end if $q$ is odd.

We now determine under what conditions complete closure of the spring occurs. The distance moved by the impact end is given by $y=v_{0} t-\frac{1}{2} a t^{2}$, whence $t=1 / a\left\{v_{0}-\right.$ $\sqrt{v_{0}^{2}-2 a y}$. Complete closure occurs if, and only if, $y$ attains the value $l \epsilon_{\max }$, i.e. if, and only if, $v_{0}^{2}-2 a l \epsilon_{\max } \geq 0$. This condition may be written in the form $2 \lambda K \leq 1$.

Closure from the fixed end. We consider here the case where closure commences from the end $X=1$ of the spring at $T=(2 k+1)+0$. Hence we have

$$
\left\{(2 k+1)-2 k^{2} \lambda\right\}<K \leq\{(2 k+2)-2 k(k+1) \lambda\}, \quad \text { and } \quad(1-2 k \lambda)>0 .
$$

The closure wave has a velocity not less than $c_{0}$ and we suppose that the wave front has reached $X=(1-\chi)$ at $T=(2 k+1)+\tau$. The configuration in the $(X, T)$-plane is shown in Fig. 5 (for the case $k=0$ ), as before the shaded portion depicting coil closure.

The closed portion of the spring $(1-\chi) \leq X \leq 1$ is at rest, so the stress throughout is constant and equal to that at $X=(1-\chi)$. Now ahead of the closure wave front 
$\Sigma=\Phi$ and Eq. (11) gives $\Delta \Sigma=\Omega^{2} \Delta \Phi$. Hence closure ceases when $\Omega=1$, the coils then barely coming into contact. $(\Omega=d \chi / d \tau)$.

Just ahead of the closure wave front we have

and

$$
\Sigma=\Phi=\left\{(2 k+1)(1-\lambda \tau)-\left(2 k^{2}+2 k+1\right) \lambda+\grave{\lambda}(1-\chi)\right\}
$$

$$
V=\{(1-\lambda \tau)-(2 k+1) \lambda x\} \text {. }
$$



FIG. 5.

Just after the wave front has passed, $\Phi=K, V=0$. Substituting in the equation $|\Delta V|=\Omega \Delta \Phi$, putting $\Omega=d \chi / d \tau$ and integrating we obtain

$$
\begin{gathered}
\lambda\left\{\chi^{2}+2(2 k+1) \chi \tau+\tau^{2}\right\}-2 \tau+2\{K-(2 k+1)+2 k(k+1) \lambda\} \chi=0 \\
(\tau=0 \quad \text { when } \quad x=0) .
\end{gathered}
$$

Equation (13) is that of a conic in the $(X, T)$-plane. If $k=0$ the conic is a parabola and if $k \geq 1$ a hyperbola.

Case $k=0$. Closure commences at $T=1+0,1<K \leq 2$. The equation of the parabola is

$$
\lambda(\chi+\tau)^{2}-2 \tau+2(K-1) \chi=0 .
$$

This may be written in the form

$$
\lambda\left\{U-\frac{(2-K)}{2 \sqrt{2} \lambda}\right\}^{2}=\frac{K}{\sqrt{2}}\left\{V+\frac{(2-K)^{2}}{4 K \lambda \sqrt{2}}\right\}
$$

where

$$
U=\frac{1}{\sqrt{2}}(x+\tau), \quad V=\frac{1}{\sqrt{2}}(\tau-\chi) .
$$

The parabola is shown in Fig. 6, with $K<2$. Its axis is parallel to the $V$-axis, and its vertex $A$ is at $U=(2-K) / 2 \sqrt{2} \lambda, V=-(2-K)^{2} / 4 \sqrt{2} K \lambda$. The tangent to the parabola at $A$ is parallel to the $U$-axis and so at $A$ we have $d \chi / d \tau=1$. If $\chi \geq 1$ at $A$ then complete closure occurs, while if $\chi<1$ only partial closure occurs. But at $A$

$$
x=\frac{1}{\sqrt{2}}(U-V)=\left(4-K^{2}\right) / 8 K \lambda \text {. }
$$


Hence if $K^{2} \leq 4(1-2 \lambda K)$ complete closure of the spring occurs on this closure wave, while only partial closure occurs if $K^{2}>4(1-2 \lambda K)$. Now $1<K \leq 2$. Thus we obtain the necessary and sufficient conditions for complete closure in the form

$$
\left\{\begin{array} { l } 
{ 0 \leq \lambda < 3 / 8 } \\
{ 1 < K \leq 2 \{ \sqrt { 4 \lambda ^ { 2 } + 1 } - 2 \lambda \} }
\end{array} \quad \text { or } \quad \left\{\begin{array}{l}
1<K \leq 2 \\
\lambda \leq\left(4-K^{2}\right) / 8 K
\end{array}\right.\right.
$$

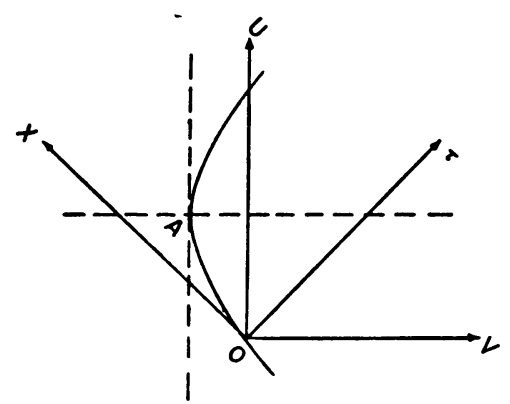

FIG. 6.

The case $\lambda=0$ is included here and it is seen that if $K=2$ this is the only case in which complete closure can take place. From Eq. (14), $\{1-\lambda(\chi+\tau)\}=+\sqrt{1-2 \lambda K \chi}$, since $\tau=0$ when $\chi=0$. The stress behind the closure wave $(1-\chi \leq X \leq 1)$ is

$$
\Sigma=\frac{K\{1-\lambda(\chi+\tau)\}}{[K-\{1-\lambda(\chi+\tau)\}]}=\frac{K \sqrt{1-2 \lambda K \chi}}{\{K-\sqrt{1-2 \lambda K \chi}\}} .
$$

As $\chi$ increases this decreases. We also find that

$$
\Omega=\frac{d \chi}{d \tau}=\frac{\sqrt{1-2 \lambda K \chi}}{\{K-\sqrt{1-2 \lambda K \chi}\}} .
$$

The case of partial closure after the first wave has been considered. Here $1<K \leq 2$, $K^{2}+8 \lambda K-4>0$. Closure ceases when

$$
\chi=\frac{\left(4-K^{2}\right)}{8 \lambda K}=\chi^{*} \quad \text { and } \quad \tau=\frac{(3 K-2)(2-K)}{8 \lambda K}=\tau^{*} .
$$

It may be shown that the spring then starts opening up at the constant rate $c_{0}$ (relative to the unstrained spring). If, moreover, closure does not commence from the impact end when the discontinuity wave is reflected there, then no more closures occur and the configuration in the $(X, T)$-plane is as shown in Fig. 7. The condition for no further closure is $K^{2}-8 \lambda K+4 \leq 0$, so together with the above conditions we have

$$
\left\{\begin{array} { l } 
{ \frac { 1 } { 2 } \leq \lambda < 5 / 8 } \\
{ 2 \{ 2 \lambda - \sqrt { 4 \lambda ^ { 2 } - 1 } \} \leq K \leq 2 }
\end{array} \text { or } \left\{\begin{array} { l } 
{ \lambda \geq 5 / 8 } \\
{ 1 < K \leq 2 , }
\end{array} \text { or } \quad \left\{\begin{array}{l}
1<K \leq 2 \\
\lambda \geq \frac{4+K^{2}}{8 K} .
\end{array}\right.\right.\right.
$$

If $K^{2}>4|1-2 \lambda K|$, then partial closure occurs from the fixed end after the first wave and closure commences from the impact end after the second wave. Complete closure may, or may not, eventually occur (according as $2 \lambda K \leq 1$, or $2 \lambda K>1$, respectively). 




FIG. 7 .

Closure from the impact end. We consider here the case in which closure commences from the end $X=0$ of the spring at time $T=2 k+0,(k \geq 0)$. Hence we have

$$
2 k\{1-(k-1) \lambda\}<K \leq\left\{(2 k+1)-2 k^{2} \lambda\right\}, \quad \text { and } \quad(1-2 k \lambda)>0 .
$$

The closure wave has a velocity not less than $c_{0}$ and we suppose that the wave front has reached $X=\chi$ at $T=2 k+\tau$. The closed portion of the coil is moving as a rigid body with the velocity of the impact end and hence it is being decelerated at a constant rate. The configuration in the $(X, T)$-plane is shown in Figs. 8 and 9 for the cases $k=0$, $k=1$, respectively.

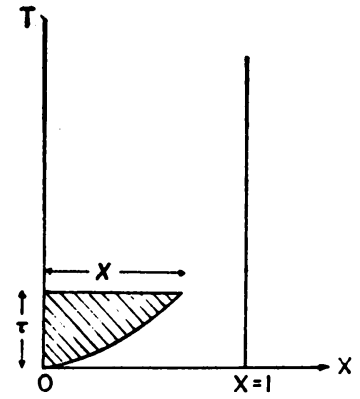

FIG. 8.

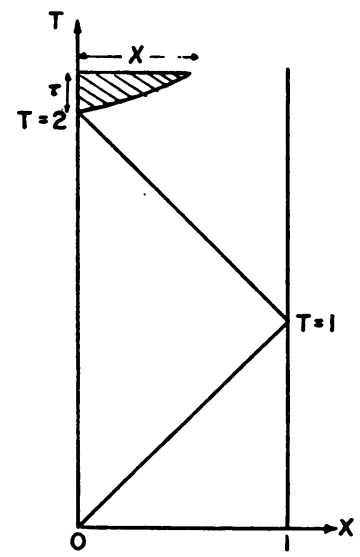

FIG. 9.

By use of Eq. (11) we find $\chi, \tau$ are connected by the relation

$$
\lambda \tau^{2}+4 k \lambda \chi \tau+2\{K-2 k(1-k \lambda)\} \chi-2 \tau=0 .
$$

If $k=0$ this is the equation of a parabola and if $k \geq 1$ that of a hyperbola. We consider the case $k=0$. 
Since the portion $0 \leq X \leq \chi$ is moving as a rigid body with uniform retardation $a$ we obtain

$$
\Sigma=\left.\Sigma\right|_{X=x}-\lambda(\chi-X), \quad 0 \leq X \leq \chi .
$$

Hence the stress in the closed portion decreases as $X$ decreases. From Eq. (11) we find

$$
\left.\Sigma\right|_{X=\chi}=\frac{1}{K}(1-\lambda \tau)^{2}=\frac{1}{K}(1-2 \lambda K \chi),
$$

using Eq. (17). Hence at a pt. in the closed portion $\Sigma$ decreases as $\chi$ increases. Complete closure takes place if, and only if, $\left(K^{2}+3 \lambda K-1\right) \leq 0$. We also have the condition $0<K \leq 1$. Hence we obtain the necessary and sufficient conditions for complete closure in the form

$$
\left\{\begin{array} { l } 
{ \lambda \geq 0 } \\
{ 0 < K \leq \frac { 1 } { 2 } \{ \sqrt { 9 \lambda ^ { 2 } + 4 } - 3 \lambda \} , }
\end{array} \text { or } \quad \left\{\begin{array}{l}
0<K \leq 1 \\
0 \leq \lambda \leq \frac{(1-K)}{3 K} .
\end{array}\right.\right.
$$

4. Mass attached to impact end of spring. In this section we consider the case in which a mass $M$ is attached to the end $x=0$ of the spring. The spring is initially at rest and unstrained, the axis of the spring being horizontal, when the attached mass is given an impulsive velocity $v_{0}$ at time $t=0$.

The equation of motion for the mass $M$ is

$$
\frac{d W}{d T}=-\mu S,
$$

where $W=\left.V\right|_{x=0}, S=\left.\Sigma\right|_{x=0}$ and $\mu=m l / M$. Until closure occurs the configuration in the $(X, T)$-plane is as shown in Fig. 3. A wave of velocity, stress and strain discontinuity is propagated along the spring with constant speed $c_{0}$, commencing at the end $x=0$ at time $t=0$ and being reflected alternately at the ends.

Using Eqs. (4), (5) and (20), we find

In region $0: \Sigma=V=0$

In region 1: $\Sigma=V=e^{-\mu(T-X)}$

In region 2: $\Sigma=2 e^{-\mu(T-1)} \cosh \{\mu(X-1)\}$,

$$
V=2 e^{-\mu(T-1)} \sinh \{\mu(X-1)\} .
$$

Since, in region $1, T \geq X$, we have $\Sigma \leq 1$. ( $\mu>0$.) Hence, if $0<K \leq 1$ closure commences from the impact end immediately and if $K>1$ no closures occur in $0 \leq T<1$. For $1 \leq T<2$, if $1<K \leq 2$ closure commences from the fixed end at $T=1$ and if $K>2$ no closures occur. If $2<K \leq\left(2+e^{-2 \mu}\right)$ closure commences from the gun end at $T=2$. We consider these three closures in detail.

Closure from fixed end after first wave. $1<K \leq 2$. Closure commences at $T=1$. We suppose that at $T=1+\tau$ the closure wave has moved along the spring to $X=$ 
$(1-\chi)$ the portion $(1-\chi) \leq X \leq 1$ being closed and at rest. The configuration in the $(X, T)$-plane is as shown in Fig. 5. Now just ahead of the closure wave front we have $\Phi=V=\Sigma=e^{-\mu(\tau+x)}$. Setting $\Omega=d \chi / d \tau$ we obtain from Eq. (11)

$$
e^{-\mu(\tau+x)}=\frac{d \chi}{d \tau}\left\{K-e^{-\mu(\tau+x)}\right\} .
$$

Integrating,

$$
K \mu \chi+e^{-\mu(x+r)}=1
$$

since initially $\chi=0=\tau$. Hence

$$
\tau=\frac{1}{\mu} \log \left\{\frac{1}{1-K \mu \chi}\right\}-\chi .
$$

Also, $\Omega=(1-K \mu \chi) /\{(K-1)+K \mu \chi\}$ which decreases as $\chi$ increases. We find $\Sigma=K(1-K \mu \chi) /\{(K-1)+K \mu \chi\}$, for $(1-\chi) \leq X \leq 1$. Complete closure occurs on this wave if, and only if, $(1-K \mu) \geq\{(K-1)+K \mu\}$, i.e., $K(1+2 \mu) \leq 2$. But $1<K \leq 2$. Therefore,

$$
\left\{\begin{array} { l } 
{ 1 < K \leq 2 } \\
{ \mu \leq \frac { ( 2 - K ) } { 2 K } }
\end{array} \text { or } \quad \left\{\begin{array}{l}
0 \leq \mu<1 / 2 \\
1<K \leq \frac{2}{(1+2 \mu)}
\end{array}\right.\right.
$$

If complete closure occurs then the time taken is given by $(1+\tau)=1 / \mu \log (1 /(1-$ $\left.\left.K_{\mu}\right)\right)=T^{\prime}$. But during the period $0 \leq T \leq T^{\prime}, W=e^{-\mu T}$. Therefore $\int_{0}^{T^{\prime}} W d T=K$ so that the gun end has moved through a distance $l \epsilon_{\max }$, which is correct.

If complete closure does not occur then closure ceases when $\Omega=1$, i.e., when $\chi=$ $(2-K) / 2 K \mu=\chi^{*}$ and then $\tau=1 / 2 \mu\{2 \log (2 / K)+1-(2 / K)\}=\tau^{*}$.

Closure from impact end at $T=2 k(k \geq 0)$. Let us suppose that closure commences (for the first time) at the impact end at $T=2 k$. With the usual notation put $T=2 k+\tau$, and let $X=\chi$ be the position of the closure wave front. Let the stress and velocity just ahead of the closure wavefront be given by $\Phi=\Sigma=\Sigma(\chi, \tau), V=V(\chi, \tau)$. Since no previous closures have occurred Eq. (3) holds in this region ahead of the closure wavefront. Hence we obtain

$$
\frac{\partial V}{\partial \tau}+\frac{\partial \Sigma}{\partial \chi}=0, \quad \frac{\partial \Sigma}{\partial \tau}+\frac{\partial V}{\partial \chi}=0 .
$$

$W=W(\tau)$ gives the velocity of the impact end and $S=\left.\Sigma\right|_{X=0}$. From Eq. (11) we find

$$
\{W-V(\chi, \tau)\}=\frac{d \chi}{d \tau}\{K-\Sigma(\chi, \tau)\} .
$$

and

$$
\left.\Sigma\right|_{x-\chi}=\Sigma(\chi, \tau)+\frac{d \chi}{d \tau}\{W-V(\chi, \tau)\}
$$


But, since the portion $0 \leq X \leq x$ is closed and moving as a rigid body,

$$
\Sigma=\Sigma:_{x=x}+(\chi-X) \frac{d W}{d \tau}, \quad 0 \leq X \leq \chi
$$

and in particular

$$
S=\left.\Sigma\right|_{X=x}+\chi \frac{d W}{d \tau}
$$

Hence

$$
S=\left(\chi \frac{d W}{d \tau}+W \frac{d \chi}{d \tau}\right)+\left\{\Sigma(\chi, \tau)-\frac{d \chi}{d \tau} V(\chi, \tau)\right\} .
$$

But, from Eq. (20), $d W / d \tau+\mu S=0$. Hence

$$
\frac{d}{d \tau}\{W(1+\mu \chi)\}+\mu\left\{\Sigma(\chi, \tau)-V(\chi, \tau) \frac{d \chi}{d \tau}\right\}=0 .
$$

Let $F(\chi, \tau)$ satisfy the conditions

$$
-\frac{\partial F}{\partial \tau}=\mu \Sigma, \quad \frac{\partial F}{\partial \chi}=\mu V, \quad F(0,0)=W(0),
$$

in virtue of the equation $\partial V / \partial \tau+\partial \Sigma / \partial \chi=0$.

Then we obtain on integration

$$
W(1+u \chi)=F(\chi, \tau) .
$$

Substituting for $W$ from Eq. (23) we find that

$$
(1+\mu \chi)\{K-\Sigma(\chi, \tau)\} \frac{d \chi}{d \tau}+\{(1+\mu \chi) \cdot V(\chi, \tau)-F(\chi, \tau)\}=0 .
$$

But

$$
\begin{aligned}
& \frac{\partial}{\partial \tau}[(1+\mu \chi)\{K-\Sigma(\chi, \tau)\}]-\frac{\partial}{\partial \chi}\{(1+\mu \chi) \cdot V(\chi, \tau)-F(\chi, \tau)\} \\
&=-(1+\mu \chi) \cdot \frac{\partial \Sigma}{\partial \tau}-\mu V+\frac{\partial F}{\partial \chi}-(1+\mu \chi) \frac{\partial V}{\partial \chi} \\
&=\left(\frac{\partial F}{\partial \chi}-\mu V\right)-(1+\mu \chi) \cdot\left(\frac{\partial \Sigma}{\partial \tau}+\frac{\partial V}{\partial \chi}\right)=0 .
\end{aligned}
$$

Hence the differential equation integrates immediately.

Case $k=0.0<K \leq 1$. Closure commences from the impact end immediately. The configuration in the $(X, T)$-plane is as shown in Fig. 8. Here $\Sigma(\chi, \tau)=V(\chi, \tau)=0$, and $W(0)=1$. Therefore $F(\chi, \tau)=1$. Hence, from Eq. (25), $W(1+\mu \chi)=1$ and from Eq. (26), $K(1+\mu \chi) d \chi / d \tau=1$. Therefore $K \chi(2+\mu \chi)=2 \tau$, since $\chi=0$ when $\tau=0$. 
This is the equation of a parabola whose axis is parallel to the $T$-axis. We find that

$$
\Omega=\frac{d \chi}{d \tau}=\frac{1}{K(1+\mu \chi)} \quad \text { and } \quad S=\frac{1}{K(1+\mu \chi)^{3}} .
$$

Also we have

$$
\Sigma=\frac{(1+\mu X)}{K(1+\mu \chi)^{3}}, \quad 0 \leq X \leq \chi .
$$

Hence in the closed portion $\Sigma$ decreases as $X$ decreases so that the coils remain in contact provided $S \geq K$. As $\chi$ increases $S$ decreases so complete closure takes place if, and only if,

$$
\left.S\right|_{x=1} \geq K, \quad \text { i.e. } \quad K^{2}(1+\mu)^{3} \leq 1 . \quad \text { but } \quad 0<K \leq 1 .
$$

Therefore,

$$
\left\{\begin{array} { l } 
{ \mu \geq 0 } \\
{ 0 < K \leq ( 1 + \mu ) ^ { - 3 / 2 } }
\end{array} \text { or } \quad \left\{\begin{array}{l}
0<K \leq 1 \\
0 \leq \mu \leq\left(K^{-2 / 3}-1\right) .
\end{array}\right.\right.
$$

If this condition is not satisfied the coils start to open up from the end $X=0$ when

$$
\chi=\frac{1}{\mu}\left(K^{-2 / 3}-1\right)=\chi^{*}
$$

and then

$$
\tau=\frac{K}{2 \mu}\left(K^{-4 / 3}-1\right)=\tau^{*} .
$$

Case $k=1 . \quad 2<K \leq\left(2+e^{-2 \mu}\right)$.

The configuration in the $(X, T)$-plane is as shown in Fig. 9. Here

$$
\begin{aligned}
& \Sigma(\chi, \tau)=2 e^{-\mu(\tau+1)} \cosh \{\mu(1-\chi)\}, \\
& V(\chi, \tau)=-2 e^{-\mu(\tau+1)} \sinh \{\mu(1-\chi)\} \quad \text { and } \quad W(0)=e^{-2 \mu} .
\end{aligned}
$$

Hence, from Eq. (25),

$$
W(1+\mu \chi)=2 e^{-\mu(\tau+1)} \cosh \{\mu(1-\chi)\}-1 .
$$

Also, from Eq. (26), we obtain on integration

$$
K \mu \chi(2+\mu \chi)+2 \mu \tau=4\left\{1-e^{-\mu(1+\tau)}[(1+\mu \chi) \sinh \{\mu(1-\chi)\}+\cosh \{\mu(1-\chi)\}]\right\} \text {. }
$$

5. Discussion of results obtained. This paper has been devoted to investigating the occurence of coil closure and the stresses during closure in a helical compression spring one end of which is fixed, the other being given an impulsive velocity when the spring is initially unstrained and at rest. The number $K=1 / v_{0}\left(c_{0} \epsilon_{\max }\right)$ arises, where $v_{0}$ is the impact velocity and $c_{0} \epsilon_{\max }$ is a quantity depending on the dimensions and material of the spring. Inelastic coil on coil impact conditions have been assumed so that the coils . remain in contact after closure, until separated by the elasticity of the spring. The results may, however, give a good indication as to the actual state of affairs in which bouncing of the coils occurs. 
In the first instance the case in which the impulsive velocity at the impact end was maintained was considered. Here complete closure inevitably occurs and it was found that exceptionally high stresses could be set up during closure if the coils were very close together prior to the passage of the closure wave. Closure commences from either the impact or the fixed end and complete closure occurs on that wave.

Secondly, the case in which the impact end was retarded at a constant rate $a$ was considered. The number $\lambda=\left(a / v_{0}\right)\left(l / c_{0}\right)$ occurs, where $l / c_{0}$ is a quantity depending on the dimensions and the material of the spring. The criterion for complete closure is $2 \lambda K \leq 1$, so that if complete closure of the spring is to be avoided we need $\left(2 a / v_{0}\right) l \epsilon_{\max }>1$. Note that $l \epsilon_{\max }$ depends on the dimensions of the spring only and not on the material. Thus it is seen that, unlike the first case, complete closure does not necessarily occur and for a given spring and prescribed impact velocity it will not occur if the retardation of the impact end is sufficiently large. Further, it is found that it is possible that no closure whatsoever will occur but that if closure does occur it commences from an end of the spring. If $0<K \leq 1$ closure commences immediately from the impact end, whilst if $1<K \leq 2$ closure commences from the fixed end after the passage of the first discontinuity wave, and these statements are independent of the value of $\lambda$. For $K>2$, no closure occurs if $\lambda \geq 1 / 2$. If $1<K \leq 2$ and only partial closure occurs from the fixed end after the first wave, then, if closure does not commence from the impact end after the second wave, no more closures occur at all. However, if closure does start from the impact end after the second wave complete closure of the spring does not necessarily occur.

Lastly, the case in which there was a mass attached to the impact end was considered. The number $\mu$, which is equal to the ratio of the total weight of the spring to the weight of the attached mass, occurs. This will, in general, be small. If $0<K \leq 1$ closure commences immediately from the impact end and unless $K$ is fairly close to 1 complete closure will occur on this wave. If $1<K \leq 2$ closure commences from the fixed end after the first wave and complete closure will occur on this wave unless $K$ is fairly close to 2 ( $\mu$ being small). However, if $\mu \geq 1 / 2$, complete closure will not occur on this wave, however close $K$ is to 1 . It is noted that $\mu=0$ in this case and $\lambda=0$ in the second case both correspond to the first case.

We will now discuss the stresses in the spring. Before closure occurs the stress, or nominal compressive force, is proportional to the nominal compressive strain and less than the quantity $m c_{0} v_{0} K$. When, however, coils of the spring come into contact, and remain so, the compressive force is equal to $m c_{0} v_{0} \Sigma$ where $\Sigma>K$. In the first case the compressive force in the closed portion of the spring remains constant while closure is taking place and, as previously mentioned, we may have a large compressive force. The time taken for the closure wave to traverse the length of the spring is correspondingly small, though. In the other two cases the value of $\Sigma$ does not remain constant in the closed portion of the spring while closure is taking place. When closure commences from the fixed end $\Sigma$ is the same throughout the closed portion at any given instant, but it decreases as the closure wavefront passes only the spring. When closure commences from the impact end the value of $\Sigma$ at a given position in the closed portion of the spring again decreases as the closure wavefront passes along the spring. In addition, however, the value of $\Sigma$ decreases uniformly as we pass along the spring from just behind the closure wavefront to the impact end, this uniform rate of decrease being independent, of the time in the second case. 


\section{APPENDIX}



Acknowledgement. The author wishes to thank Professor E. H. Lee for his advice in the preparation of this paper.

\section{BIBLIOGRAPHY}

1. E. H. Lee, Wave propagation in helical compression springs, Proceedings of 5th Symposium of Applied Mathematics of the American Mathematical Society, June 1952. (To be published by McGraw-Hill). 